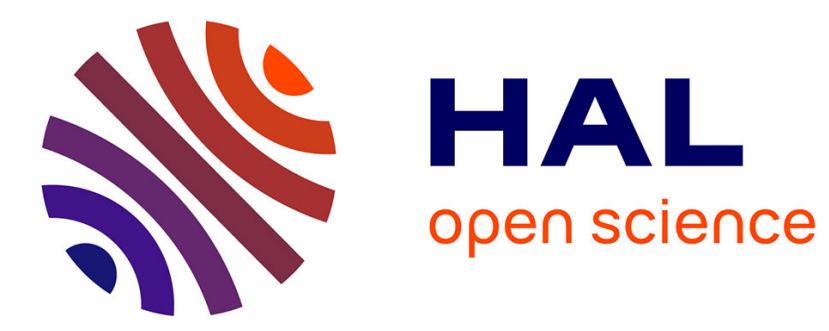

\title{
Spreading of porous vesicles subjected to osmotic shocks: the role of aquaporins
}

Alice Berthaud, François Quemeneur, Maxime Deforet, Patricia Bassereau, Françoise Brochard-Wyart, Stéphanie Mangenot

\section{To cite this version:}

Alice Berthaud, François Quemeneur, Maxime Deforet, Patricia Bassereau, Françoise Brochard-Wyart, et al.. Spreading of porous vesicles subjected to osmotic shocks: the role of aquaporins. Soft Matter, 2016, 12 (5), pp.1601-1609. 10.1039/C5SM01654A . hal-01251442

\section{HAL Id: hal-01251442 https://hal.sorbonne-universite.fr/hal-01251442}

Submitted on 6 Jan 2016

HAL is a multi-disciplinary open access archive for the deposit and dissemination of scientific research documents, whether they are published or not. The documents may come from teaching and research institutions in France or abroad, or from public or private research centers.
L'archive ouverte pluridisciplinaire $\mathbf{H A L}$, est destinée au dépôt et à la diffusion de documents scientifiques de niveau recherche, publiés ou non, émanant des établissements d'enseignement et de recherche français ou étrangers, des laboratoires publics ou privés. 


\title{
Spreading of porous vesicles submitted to osmotic shocks: The role of aquaporins
}

\author{
Alice BERTHAUD ${ }^{*}$, François QUEMENEUR ${ }^{* a}$, Maxime DEFORET ${ }^{\mathrm{a}}$, Patricia \\ BASSEREAU $^{\mathrm{a}}$, Françoise BROCHARD-WYART ${ }^{\mathrm{a}}$, Stéphanie MANGENOT ${ }^{\mathrm{a}}$
}

\footnotetext{
${ }^{a}$ Sorbonne Université, UPMC Univ Paris 06, CNRS, UMR 168, Institut Curie, 26 rue d'Ulm, 75248 Paris Cedex 05, France.

* Both authors have equally contributed

CORRESPONDENCE To: Stéphanie Mangenot, Laboratoire Physico-Chimie, Centre de Recherche de I'Institut Curie, 26 Rue d'Ulm, 75248 Paris, France Tel: +33156246460, Fax: +331405106 36,

E-mail: stephanie.mangenot@curie.fr.

Electronic Supplementary Information (ESI) available
}

\section{Glossary:}

$C_{i}, C_{f}$ : Initial and final sucrose concentration in GUV

$V_{i}, V_{f}:$ Initial and final GUV volume

$\boldsymbol{n}$ : Number of sucrose molecules in the GUVs

$\Pi_{0}, \Pi_{f}:$ Osmotic pressure of glucose before and after the shock

$V_{r}$ : Reduced volume of the osmotic shock

$R(t)$ Radius of the GUV at time $t$

$A(t)$ : Area of the adhering patch at time $t$

$\boldsymbol{R}_{\text {patch }}:$ Radius of the adhering patch

$V(t)$ : Volume of the GUV at time $t$

$\boldsymbol{R}_{v 0}, \boldsymbol{R}_{v}$ : Radius of the GUV before and during adhesion

$\boldsymbol{R}_{v f}$ : Radius of the GUV at equilibrium after the osmotic shock

$A_{0}, \boldsymbol{A}_{f}$ : GUV surface before and at equilibrium after the osmotic shock

$S_{0}, S:$ Surface of the GUV before and during adhesion

$\boldsymbol{V}_{\mathbf{0}}, \boldsymbol{V}_{\boldsymbol{f}}$ : GUV volume before the shock and at equilibrium after the shock

$\gamma_{0}, \gamma$ : Surface tension of the GUV before and during adhesion

$\kappa_{d}$ : Bending modulus of the GUV membrane

$\theta$ : Contact angle between the GUV and the surface

$\theta_{c}:$ Critical contact angle for GUV spreading

$t_{c}$ : Critical time for GUV spreading

$D$ : Diffusion coefficient of the binders

$\Gamma_{i}, \Gamma_{0}$ : Binder density on the GUV inside and outside the adhering patch

$U$ : Energy gain per sticker finding a binding partner

$P_{\text {memb }}$ : Intrinsic membrane permeability

$P_{A Q P}:$ Permeability of the membrane due to aquaporins

$\tau:$ Deswelling time

$N$ : Total number of aquaporins

$R_{p}$ : Radius of the aquaporin pore

$\sigma:$ Pore density in the membrane

$\eta$ : Bulk viscosity

$v_{0}$ : Molar volume of water

$\mathrm{C}_{\mathrm{i}}, \mathrm{C}_{\mathrm{E}}$ : Internal and external concentration of solute 


\section{A INTRODUCTION}

Water transport across membranes is a fundamental property of life and is involved at cellular or subcellular level. In human body, 180 liters of primary urine per kidney have to be filtered every day. The lipid membrane alone is incapable to transport such water quantity ${ }^{1}$. The specific and fast water transport across membranes is achieved by a class of transmembrane proteins, the aquaporins. The 13 mammalian aquaporins (AQPs) are specific to cell types and tissues ${ }^{2}$. In the eyes lenses, water transport is performed by the Aquaporin-0 (AQPO) ${ }^{3}$. The AQPO is unique among the AQP family. First, it transports water at a much slower rate than the other aquaporins and secondly, they are able to form junctions between cells depending on their location in the lens 4 . Full length AQPOs are present in the young fiber cells, at the periphery of the lens. They function as water channels but with a low water conductance. As a matter of comparison, AQPO water conductance is approximately 40 fold less than AQP1, one of the most efficient water pore found in many tissues ${ }^{6}$. In the core of the lens, AQP0s are present as a mixture of full-length and truncated AQPOs. Truncated AQPOs result from the proteolytic cleavage of full AQPO at the C-terminus end during the maturation process of fiber cells. ${ }^{7}$. The capability of this truncated AQPO to conduct water is still unclear.

Water permeability across membranes has been studied for a long time by various techniques including NMR ${ }^{8}$ or isotope tracer efflux ${ }^{9}$. Alternative methods use osmotic shock to induce water flow: for instance, after aquaporin overexpression in Xenopus oocytes ${ }^{6} 10$ or purification and reconstitution in small liposome ${ }^{11}$ (SUV, radius $<100$ $\mathrm{nm}$ ). These systems are osmotically deflated by rapid mixing with a solution of higher osmolarity ${ }^{6,}{ }^{10,11}$. The difference of osmotic pressure between the two sides of the membrane results in a water efflux. The rate of volume change is directly related to the permeability of the membrane and the presence of proteins within the membrane. cRNA injection in oocytes is the most versatile method. It is indeed very easy to control precisely the sequence of the protein inserted in the membrane and no biochemical purification steps and/or extraction of native membrane are necessary. Conversely, it is very difficult to determine quantitatively the amount of proteins expressed and incorporated into the membrane. It is therefore possible to measure the global membrane permeability value, but the permeability of a single channel could only be estimated.

Another method for precisely controling the density of channels uses proteo-liposomes where membrane channels are inserted in small unilamellar vesicle. It requires a biochemical purification of the proteins but proteins can be inserted in almost all lipid mixtures. The water permeability is determined by the volume change of the liposomes subject to an osmotic shock. The volume variation is either deduced by a change of fluorescence intensity $^{12-14}$ or by dynamic light scattering ${ }^{15-19}$. In both cases, the variation is measured over an ensemble of liposomes leading to an average measurement of the water permeability. Moreover, since vesicles as well as oocytes may adopt diverse complicated shapes ${ }^{20}$, the precise determination of the volume is not obvious. Stopped-flow studies combined with quenching of a fluorescent marker encapsulated in vesicle, have been widely used to measure the permeability of the AQPs ${ }^{12-14}$.

GUV micropipette aspiration is a very accurate technique and has been widely used to measure with high precision the water permeability of membranes with various lipid compositions ${ }^{21,22}$. A GUV aspirated in a micropipette is transferred from a chamber at a low osmolarity to another one with a higher osmolarity. The deflation of the GUV due to the efflux of water leads to an increase of the length of the tongue inside the pipette. Water permeability and mechanical properties of lipid membranes can be accurately monitored with this technique. However, its main drawback is that it can only investigate a single vesicle at a time. It also requires careful coating of the interior of the pipette to avoid any interaction between the vesicle and the glass, which is 
known to be trickier in the presence of transmembrane proteins in the membrane. This might be the reason why it has never been used to measure water permeability of proteins, as far as we know.

In the present work, we propose a new method to precisely measure the water permeability of a single water channel. GUVs with AQPO reconstituted in their membrane adhere to a glass surface via biotin-streptavidin interaction. GUVs are then exposed to osmotic shocks. We measure the increase of the contact area during GUV deswelling and spreading. Our analysis is supported by a theoretical model that considers different aspects related to GUV spreading upon adhesion and deflation. In a first step, we study the specific adhesion of vesicles coated with biotin on glass substrates decorated with streptavidin. This is a specific case of adhesion where the binders are immobilized at the contact because the streptavidins are bound to the substrate and the life time of biotin streptavidin bound is a few minutes ${ }^{23}$. In previous studies ${ }^{24,25}$, the binders were considered mobile in the contact (because the links are transient) and the expansion force is due to osmotic pressure ${ }^{26}$. In our case the driving force is due to the adhesion energy of the binders. We thus extend previous theoretical work on cellular adhesion to our vesicles spreading case of immobilized links. In a second step, we describe the deswelling of adhering vesicles due to osmotic shock. In a third step, using our model of adhesion, we relate the increased of the spreading area to the decrease of the internal GUV volume. This approach allows an accurate determination of the membrane and protein water permeability. The number of AQPOs in the membrane is precisely and independently determined using fluorescence confocal microscopy ${ }^{27}$. The technique presented here does not require any micromanipulation of vesicles across experimental chambers at different osmolarities ${ }^{21,22}$ and several GUVs can be observed simultaneously. We prove the feasibility of our method by measuring water permeability of one of the less efficient water channel: the AQPO. We also demonstrate that the truncated AQPO found in the core of the eye lenses is no more a water channel.

\section{B METHODS}

\section{Purification and reconstitution of AQPO}

The non-junctional AQPOs (Wt-AQPO) were purified from the cortex of 25 sheep eyes lens ${ }^{28,29}$. Junctional AQPOs (Tr-AQPO) were obtained by chymotrypsin digestion of Wt-AQPO (See Fig. S1A for SDS-gel). Both types of AQPO in detergent (n-Octyl- $\beta$-D-Glucopyranoside, OG; Affymetrix) were labeled with one Alexa-488-maleimide molecule (Invitrogen) per water channel and reconstituted in Small Unilamellar Vesicles (SUV) of (EPC:EPA) (9:1) 75\%, 20\% cholesterol, 5\% DSPE-PEG 2000 -Biotin (1,2-distearoyl-sn-glycero-3-phosphoethanolamine- $\mathrm{N}$-[biotinyl-(polyethylene glycol)-2000) and 0.25\% Texas Red 1,2-dihexadecanoyl-sn-glycero-3-phosphoethanolamine (TR-DHPE, Invitrogen) ${ }^{27,} 30$. The maleimide group of Alexa-488 binds to the accessible thiol groups of the AQPO (ie Cys or Met). EPC, EPA, cholesterol and DSPE-PEG-Biotin were purchased from Avanti Polar Lipids. In all experiments, the lipid to protein ratio has been kept constant at 10:1 (w/w). The lipid composition was chosen to reduce the intrinsic lipid membrane permeability ${ }^{21}$.

\section{Functionalization of the glass bottom chamber}

Pre-activation of the glass cover slides was achieved by immersion in a Piranha solution (70\% sulfuric acid, $30 \%$ hydrogen peroxide) for $10 \mathrm{~min}$. After intensive rinsing with MQ water, cover slides were silanized by immersion for 2 hours in a solution of $2.86 \%(\mathrm{v} / \mathrm{v}) \mathrm{N}$-[3-(Trimethoxysilyl)propyl]-ethylenediamine, $0.86 \%(\mathrm{v} / \mathrm{v})$ acetic acid and 96.3 $\%(\mathrm{v} / \mathrm{v})$ methanol. After washing and drying for $15 \mathrm{~min}$ at $100^{\circ} \mathrm{C}$, the cover slides were then functionalized with 100 $\mu \mathrm{L}$ of a solution at $1 \mathrm{mg} \cdot \mathrm{mL}^{-1}$ of $50 \%$ metoxy- $\mathrm{PEG}_{2000}-\mathrm{NHS}$ (metoxy-PolyEthylene Glycol-N-HydroxySuccinimid; Rapp Polymere GmbH, Germany) and 50\%-PEG- 3400 -Biotin (Biotin-PolyEthylene Glycol-N-Hydroxy Succinimide; Nanocs 
NY, USA) in a carbonate-bicarbonate buffer $\mathrm{pH}$ 8.5. Streptavidin solution at $80 \mu \mathrm{M}$ (Life technologies) was then deposited on top of the biotinylated surface. One biotin over two is estimated to be grafted with one streptavidin, leading to a grafting ratio of $50 \%$.

Chambers allowing the application of successive osmotic shocks are formed by sticking circular chambers (SecureSeal (TM); Sigma Aldrich France) on top of a functionalized cover slide.

\section{Preparation of lipid vesicles and transfer}

Giant unilamellar vesicles (GUVs, $3 \mu \mathrm{m}$ < radius < $25 \mu \mathrm{m}$ ) with or without AQPO were prepared by electroformation. Proteo-lipidic mixture of SUV was deposited on indium-tin oxide (ITO) glass side, dried for $1 \mathrm{~h}$ under saturated $\mathrm{NaCl}$ vapor and rehydrated in 150 mOsm solution (sucrose and Pipes $10 \mathrm{mM} \mathrm{pH} \mathrm{6.5)} \mathrm{under} \mathrm{an}$ electric field. A sinusoidal tension of $1.1 \mathrm{~V}$ and $10 \mathrm{~Hz}$ was applied on the ITO glass sides for 1 hour to produce GUVs 27, 31 . An aliquot of $5 \mu \mathrm{L}$ of the GUV solution was transferred to an iso-osmotic solution of glucose, Pipes $10 \mathrm{mM} \mathrm{pH}$ 6.5 and $10^{-4} \mathrm{mM}$ of 6-carboxyfluorescein to check the absence of leakiness.

GUVs collected from the ITO glass were then transferred and sedimented by gravity in a chamber with functionalized glass bottom filled with $90 \mu \mathrm{L}$ of glucose $150 \mathrm{mM}, 10 \mathrm{mM}$ Pipes $\mathrm{pH}$ 6.5.

\section{Osmotic shock}

Osmotic shocks were performed by adding controlled amounts of glucose solution of suitable concentration in the external solution. The number of sucrose molecules inside the GUV is constant during the shock and equal to: $n=C_{i} V_{i}=C_{f} V_{f}$ where $V_{i}, V_{f}$ and $C_{i}, C_{f}$ are the initial and final GUV volumes and sucrose concentrations in the GUV, respectively. The shocks are characterized by their reduce volumes $V_{r}=\frac{V_{f}}{V_{i}}=\frac{C_{i}}{C_{f}}$. In our experiments, $V_{r}$ ranges from 0.98 to 0.72 .

\section{RICM experiments and fluorescence experiment}

Reflection Interference Contrast Microscopy (RICM) experiments were performed on a Nikon Eclipse Ti inverted microscope equipped with an interferometric filter and a mercury lamp (546 nm) (Nikon Intenslight) following classical procedures ${ }^{32}$. Images were recorded with a highly sensitive digital camera (iXon+, Andor Technology; Belfast UK) with an acquisition rate of 10 images per second.

Lipid and aquaporin fluorescences were measured with the same microscope equipped with an Eclipse C1 confocal line with two lasers $(\lambda=488 \mathrm{~nm}$ and $543 \mathrm{~nm}$ ) and a Nikon Plan-Fluor 100x oil objective (1.3 NA). Quantitative fluorescence analysis was performed as previously described to extract the surface density of AQPO in the GUV membrane ${ }^{27}$. RICM experiments and confocal imaging were performed alternately on the same sample. The radius of the GUV was measured prior to any osmotic shock and at equilibrium using confocal microscopy.

\section{RICM data analysis}

The patch area extraction from RICM images was performed within a home-made Matlab (MathWorks, Natick, MA) graphical-user-interface environment, available in Supporting Information (SI text) (RICM-patch-gui.m). The automated patch recognition from a sequence of RICM images is based on morpho mathematical operations and is guided by the operator. Initially, the patch in the first frame is manually segmented. Then the time-series of the patch area is generated by an iterative process: the segmented patch in the frame $i$ will support the segmentation of the patch in the frame $i+1$. The contour of the patch is detected by the watershed function applied to an image that was transformed to take into account the shape of the patch in the previous frame (using erosion, dilation, and minima imposition functions). The algorithm is efficient as long as the patch area does not grow too fast. In 
case of segmentation error, the operator stops the iterative process, manually segments the patch, and resumes the iterative process.

\section{RESULTS}

\section{Principle of the method}

The principle of the method is presented on Fig. 1 and is summarized in this paragraph. Immediately after their formation, GUVs are transferred in an iso-osmotic glucose buffer. Each GUV characterized by a radius $R_{v 0}$ and a surface tension $\gamma_{0}$, sediments on the functionalized surface composed of grafted biotinylated lipids bound to streptavidin. Adhesion between biotinylated lipids of the GUV and streptavidin on the surface leads to the formation of an adhering patch characterized by an area $A(t)$ or a radius $R_{\text {patch }}=R_{v}$. $\sin \theta$, where $\theta$ is the contact angle between the vesicle and the surface. The area of the patch increases as a function of time up to a characteristic time $t_{c}$, above which it saturates. The characteristic time $t_{c}$ depends on the radius of the GUV and on the adhesion energy. The patch size is measured experimentally using Reflection Interference Contrast Microscopy (RICM).

When GUVs have reached the final spreading state, they are subjected to a series of hyper-osmotic shocks by adding controlled amounts of a glucose solution of suitable concentration in the external solution. The osmotic

shock is characterized by the ratio $V_{r}=\frac{V_{f}}{V_{0}}=\frac{C_{0}}{C_{f}}$. Assuming an ideal law for the osmotic pressure $\Pi$ versus glucose concentration, we can also write $V_{r}=\frac{\Pi_{0}}{\Pi_{f}}$. When a succession of shocks are applied to the vesicle, they are all considered mutually independent, the final state of the $\mathrm{N}^{\text {th }}$ shock being the initial state of the $\mathrm{N}+1$ shock. The increase of the external osmotic pressure induces an efflux of water through the lipid and the water pores, causing a decrease of the volume of the GUV. The resulting excess of membrane is transferred to the adhering patch, leading to an increase of the area of the patch followed in real time by RICM.

\section{Experiments}

\section{2a Membrane characterization}

Before the addition of the AQPO, dynamic light scattering revealed that the SUV average radius was $120 \pm 30 \mathrm{~nm}$. Both types of AQPO (Wt-AQPO and Tr-AQPO) were reconstituted separately in the SUVs by the addition of $40 \mathrm{mM}$ of $\mathrm{n}$-Octyl- $\beta$-D-glucopyranoside (OG). This detergent was removed with biobeads (10 $\mathrm{mg}$ of biobeads to remove $1.17 \mathrm{mg}$ of OG (24)). Cryo-electron microscopy on the proteo-liposomes mixtures shows that the vesicles have a diameter of the order of $100 \mathrm{~nm}$, without any visible defect in the membrane (Fig. S1.B).

After the electro-formation of the GUVs, confocal microscopy analysis confirmed the unilamellarity of the GUV containing both types of AQPO (Fig. S1.C1 and D). The average diameter was found to be $15 \pm 6 \mu \mathrm{m}$ (N =130 GUVs) independently of the presence of AQPO in the membrane (Fig. S1.C3). The density of AQPO inserted in the membrane of the GUV $\sigma$ was determined by measuring fluorescence intensity using confocal microscopy (23) and found to be equal to $1000 \pm 400 \mathrm{AQPO} / \mu \mathrm{m} 2$ for Wt-AQPO and $1300 \pm 350 \mathrm{AQPO} / \mu \mathrm{m} 2$ for Tr-AQPO (Fig. S1.E). The dispersion of the number of AQPOs inserted in the GUVs is due to preparation method of the GUVs. As the initial surface fraction of AQPO in the proteo-liposomes was 1600 AQPO/ $\mu \mathrm{m} 2$, we concluded that most of the AQP0s were incorporated in the GUVs (25).

In order to precisely determine the water permeability of both types of AQP0, we first checked that the presence of the trans-membranes proteins did not perturb the lipid permeability, in particular did not induce GUV leakiness. Prior to all osmotic shock experiments, GUVs were placed in an external buffer containing $10^{-4} \mathrm{mM}$ of fluorescent 
6-carboxyfluorescein for $10 \mathrm{~min}$, whereas the interior GUV bulk was not fluorescent initially. 93\% of the GUV interiors remained devoid of fluorescence after the shock, independent of the presence or not of AQPO in the membrane. Batches containing more than $10 \%$ of leaky GUVs were not used. We thus concluded that the lipid bilayers containing or not AQPO are not leaky to molecule bigger than $376 \mathrm{Da}$ (size of the 6-carboxyfluorescein).

\section{$2 b$ Spreading of the GUVs}

Before applying any osmotic shock, GUVs containing biotinylated lipids were sedimented on the glass surface functionalized with streptavidin. Specific binding between the GUV and the surface created an adhering patch (Fig. 1C-F), which was growing as a function of time. The size of the patch at any time was determined using an automatic custom image analysis script (See Methods). Fig. 2A displays a typical time variation of the adhesion patch area of the GUV followed by RICM immediately after the initiation of the adhesion. This graph displays two regimes, for $t<t_{c}=50 \mathrm{sec}$, the area of the patch increases as $t^{0.53 \pm 0.02}$ and saturates to a plateau value above $t_{c}$. All vesicles adhered to the surface through an adhesion patch with an almost perfect disc shape, characterized by a radius $R_{\text {patch }}$. The contact angle $\theta$ between the GUV and the surface is defined with $R_{\text {patch }}=R_{v}$. $\sin \theta$ where $R_{v}$ is the apparent radius of the GUV. $R_{v}$ was measured using fluorescence confocal microscopy just prior to the osmotic shock and after the spreading of the GUV when the patch area is constant. For $t>t_{c}$, we note $\theta_{c}$ the value for $\theta$. In all our experiments we found a value of $\theta_{c}=25 \pm 4^{\circ}(\mathrm{N}=83)$. This value is independent of the GUV radius. To perform these experiments, one must control the streptavidin grafting density on the functionalized surface. If this density is too high, transitory pores can appear due to high tension on the membrane imposed by the high adhesion energy. If transitory pores are formed, the vesicle continuously leaks water out, leading to a continuous increase of the adhering patch. In the extreme case, the GUV explodes. Therefore, the constant value of the area patch for $t>t_{c}$, is the signature of the absence of transient pores. We carefully chose our experimental conditions so that no transitory pore was observed and kept these adhesion conditions constant during all sets of experiments.

\section{2c Osmotic shocks}

When GUVs have reached the final spreading state, i.e. for $t>t_{c}$ and $\theta=\theta_{c}$, they are subjected to hyper osmotic shocks. All shocks are characterized by their reduce volume $V_{r}$.

Fig. 2B displays a typical evolution of the contact area of a vesicle containing full-length AQPO subjected to a succession of osmotic shocks. After one shock, the contact area increases and reaches an equilibrium value. Each shock causes a decrease of the vesicle volume due to the water exit. The GUV radius is determined by confocal measurement before and after the shock. Next, in order to extract the permeability of the membrane and of the single pores from our data, we thus need a model to describe both: (i) the dynamics of the GUV deswelling and (ii) the resulting increase of the adhesive patch to relate our data to the permeability of single membrane pores.

\section{Models}

Our aim is to model the variation of the vesicle contact area due to the vesicle deswelling induced by an osmotic shock. In the following, we describe the three following stages:

(i) The spreading of the GUV on the functionalized surface, leading to a spontaneous growth of the patch. We will remind the dynamics of spreading of vesicles controlled by the diffusion of binders towards the adhesive patch ${ }^{24}$. We show that the vesicles spread up to a critical contact angle $\theta_{c}$. This defines the initial state before the shock (Figs. 1D and E).

(ii) The deflation of the GUV due to an osmotic shock.

(iii) The resulting increase of the spreading area (Figs. 1E and F). 


\section{3a Spreading of the GUV: Role of binder diffusion and membrane tension}

Let's first consider the case where a GUV of radius $R_{v}$ with a small surface tension $\gamma_{0}$, coated with binder of type A, spreads on a surface functionalized with the complementary specific binder B to make an AB link (Figs. $1 A$ and $B$ ). The spreading vesicle is characterized by the size of the adhesive patch $R_{\text {patch }}$. Assuming that the volume remains constant during the spreading and that the shape is a spherical cap with a contact angle $\theta$, the increase of the surface can be written as $\frac{S-S_{0}}{S_{0}}=\frac{\theta^{4}}{16}$ where $S_{0}=4 \pi R_{v}^{2}$ is the surface of the spherical vesicle before the spreading. According to Helfrich, the increase of the GUV surface area leads to an increase of the membrane tension $\gamma$ : $\frac{S-S_{0}}{S_{0}}=\frac{k T}{8 \pi \kappa_{d}} \ln \left(\frac{\gamma}{\gamma_{0}}\right)^{33}$ where $\kappa_{d}$ is the bending modulus, and $k T$ the thermal energy. Typical values of $\kappa_{d}$, which depends on lipid composition, are around 10-20 kT. The variation of GUV surface can be rewritten as a variation of the contact angle:

$$
\frac{\theta^{4}}{16}=\frac{\theta_{c}^{4}}{16} \ln \left(\frac{\gamma}{\gamma_{0}}\right)
$$

With $\theta_{c}=\left(\frac{2 k T}{\pi \kappa_{d}}\right)^{1 / 4}$ is the critical angle of spreading.

Eq. [1] shows that if $\theta<\theta_{c}$, the surface tension $\gamma$ is almost constant, whereas if $\theta \sim \theta_{c}$ the surface tension increases exponentially with $\gamma$. As a consequence two spreading regimes can be observed depending on the $\theta$ value:

(i) For $\theta<\theta_{c}$

The surface tension is constant during the growth of the adhering patch. The spreading of the GUV is governed by adhesion. We supposed here that the GUV radius is constant during the spreading. $\Gamma_{i}$ and $\Gamma_{0}$ denote the binder density on the GUV inside and outside the adhering patch, respectively (Fig. 1B). The binders diffuse towards the adhesive patch with a diffusion coefficient $D$. We assume that at time $t$ the binders come from a region of size $\sqrt{D t}$. Assuming that the binders are mobile in the contact, the conservation of the binder numbers leads to:

$$
\left(\Gamma_{i}-\Gamma_{0}\right) \simeq \Gamma_{0} \frac{D t}{R_{\text {patch }}^{2}}
$$

In quasi static conditions, the force balance at the contact line can be written as:

$$
\frac{1}{2} \gamma_{0} \theta^{2}=\Gamma_{i} . U
$$

where $U$ is the energy gain per sticker finding a binding partner. Notice that Eq. [3] holds for immobilized stickers. As soon as a biotin reaches a streptavidin in the adhesion patch, it binds and cannot move anymore. This is different for mobile stickers discussed in previous publication ${ }^{24}$. Introducing $\varepsilon=\frac{2 U \Gamma_{0}}{\gamma_{0}}$ and combining Eqs. [2] and [3] gives the variation of the adhering patch as a function of time:

$$
R_{\text {Patch }}^{4}=\varepsilon D t R_{v}^{2}
$$

We thus expect that the area of the adhering patch varies as $v t$. In this regime, the tension is nearly constant, and the kinetics of spreading is governed by the diffusion of the binders towards the adhesive patch.

$$
\text { (ii) For } \theta \geq \theta_{c}
$$

As soon as $\theta$ reaches to $\theta_{c}$, the surface tension $\gamma$ increases exponentially according to Eq. [1]:

$$
\gamma=\gamma_{0} \exp \left(\frac{\theta}{\theta_{c}}\right)^{4}
$$

The Laplace's equation becomes:

$$
\frac{1}{2} \gamma_{0} \theta^{2} \exp \left(\frac{\theta}{\theta_{c}}\right)^{4}=\Gamma_{i} k T
$$

Combination of Eqs. [2] and [6] leads to the variation of the contact angle:

$$
\theta=\theta_{c}\left[\ln \left(t / t_{c}\right]^{1 / 4}\right.
$$


where $t_{c}=\frac{R^{2} \theta_{c}^{4}}{\varepsilon D}$ is the crossover time between the two regimes. As a consequence of the exponential growth of $\gamma$, $\theta$ is almost independent of time and closed to $\theta_{c}$. The spreading area remains nearly constant in time.

In conclusion, $A(t)$ obeys a diffusion law up to a spreading time $t_{c}=\frac{R^{2} \theta_{c}^{4}}{\varepsilon D}$ above which the spreading stops.

\section{$3 b$ Deflation}

When GUVs have reached the final spreading state, i.e. for $\theta \sim \theta_{c}$, they are subjected to hyper osmotic shocks. The shock causes a decrease of the vesicle volume due to the water exit. The deswelling of the vesicle of volume $V(t)$ and surface $S$ can be written as:

$$
\frac{d V}{d t}=-\left[S P_{m e m b} v_{o}+\frac{N r_{p}^{3} k T}{\eta}\right] n_{i}\left(\frac{1}{V_{f}}-\frac{1}{V}\right)
$$

where $P_{\text {memb }}$ is the intrinsic membrane permeability (in $\mu \mathrm{m} \cdot \mathrm{sec}^{-1}$ ) ${ }^{22}, N$ the total number of aquaporins in the GUV, $r_{p}$ the pore radius, $\eta$ the bulk viscosity and $v_{0}$ the molar volume of water. $n$ is the fixed number of solute molecules inside the vesicle, $C_{E}-C_{i}=\frac{n}{V_{f}}-\frac{n}{V}$ is the difference between external $\left(C_{E}\right)$ and internal $\left(C_{i}\right)$ concentrations at time $t=0$. Eq. [8] can also be written as:

$$
\frac{d V}{d t}=-\left[P_{m e m b}+P_{A Q P 0}\right] S v_{0} n\left(\frac{1}{V_{f}}-\frac{1}{V}\right)
$$

Where $P_{A Q P}=\frac{\sigma r_{p}^{3}}{\eta v_{0}} k T$ is the permeability of the membrane due to the aquaporins and $\sigma$ the density of pore in the membrane.

Resolution of Eq. 9 leads to

$$
\frac{V(t)-V_{f}}{V_{0}-V_{f}}=e^{-t / \tau}
$$

with $\frac{1}{\tau}=\left(P_{m e m b}+P_{A Q P 0}\right) S v_{0} \frac{C_{E}^{2}}{n_{i}}$

Note that the deswelling time $\tau$ varies as $C_{E}^{-2}$.

\section{3c Spreading due to water leak out through pores}

We have now to relate the patch area $A(t)$ to the volume $V(t)$ of the vesicle. During the shock, $R(t)$ decreases from $R_{v 0}$ to $R_{v f}$. The deswelling gives rise to an increase of the excess area. Because the vesicle is in contact with an adhesive substrate, we assume that the excess area spreads to maintain the surface tension constant. This assumption is valid if the characteristic spreading time $t_{c}$ is much faster than the deswelling time $\tau$. It leads to a conservation of the surface:

$$
S=4 \pi R_{v}(t)^{2}\left(1+\frac{\theta^{4}}{16}\right)=4 \pi R_{v 0}^{4}\left(1+\frac{\theta_{c}^{4}}{16}\right)
$$

where $R_{v}(t)$ is the radius of the sphere (see Fig. 1C) defined by $V(t)=\frac{4}{3} \pi R_{v}^{3}(t)$.

We write Eq. 11 in term of $A(t)=\pi \theta^{2} R_{v}^{2}$ at time $t$ and in term of $A_{f}$ at infinite equilibrium time, which leads to:

$$
\left\{\begin{array}{c}
A(t)^{2}-A_{0}^{2} \frac{R_{v}^{2}(t)}{R_{0}^{2}}=16 \pi^{2} R_{v}(t)^{2}\left(R_{v 0}^{2}-R_{v}(t)^{2}\right) \\
A_{f}^{2}-A_{0}^{2} \frac{R_{v f}^{2}}{R_{0}^{2}}=16 \pi^{2} R_{f}^{2}\left(R_{v 0}^{2}-R_{v f}^{2}\right)
\end{array}\right.
$$

Eqs. [10] and [12] lead to the spreading law for $A(t)$. They could be solved numerically (Fig. S2), and analytically in the limit of small shocks assuming $\frac{R_{v 0}-R_{v f}}{R_{v f}} \ll 1$, leading to:

$$
\frac{A(t)-A_{f}}{A_{0}-A_{f}}=e^{-t / \tau}
$$


Fit of Eq. [13] combined with Eq. [10] allows for the determination of the total membrane permeability $P_{\text {memb }}$. $P_{\text {memb }}$ is measured separately in the absence of pores and we can then deduce the permeability of aquaporin $P_{A Q P O}$.

\section{ANALYSIS AND DISCUSSION}

\section{Membrane spreading and characteristic time}

Before applying an osmotic shock, the kinetics of spreading is only governed by the adhering conditions (i.e. concentrations of biotin on the GUV and streptavidin on the surface). For $t<t_{c}$, the spreading area of the vesicle as a function of time is fitted by a power law with an exponent $n=0.53 \pm 0.02(\mathrm{~N}=6)$ (continuous line on Fig. $2 \mathrm{~A})$ in agreement with the theoretical model. Similar experiments on the spreading of GUV on a functionalized surface were previously reported ${ }^{32}$. In this work, GUVs were coated with streptavidin and sedimented onto biotinylated surface. Surprisingly, the spreading patch area varied with an exponent $n=0.96 \pm 0.12$. The only difference between these two experiments is that in our case, the GUVs are functionalized with biotin whereas it was with streptavidin in the other case. It is remarkable to note that two complementary experiments (i.e. biotin on GUVs or on the surface) lead to two completely different regimes of spreading. Biotins are much smaller than streptavidin proteins (52.8 Da compared with $244 \mathrm{kDa}$ ) and one streptavidin can bind 4 biotins. It explains why small biotins are able to freely diffuse and equilibrate in the adhering patch. On the other hand, when the vesicle is coated with streptavidin, the density of streptavidin accumulating in the patch is assumed to be the saturation $\Gamma_{\text {sat }}$ density. Eq. [2] with $\Gamma_{i}=\Gamma_{\text {sat }}$ leads to $\Gamma_{s} R_{\text {patch }}^{2}=\Gamma$. Dt. The spread area increases linearly in time as observed in ${ }^{32,34,35}$.

The critical angle of spreading $\theta_{c}=\left(\frac{2 k T}{\pi \kappa_{d}}\right)^{1 / 4}$ was found to be equal to $25 \pm 4^{\circ}$. We can thus derive the bending rigidity of the membrane $\kappa_{d}=18 \pm 3 k T$. The bending rigidity is known to depend on the membrane composition. It varies between 10 to $30 k T^{36}$ for GUV containing only phosphatidylcholine (PC) membrane with chain lengths ranging from 18 to 22 carbons and with different degrees of saturation. In the present work, we used a mixture of 75\% (EPC:EPA) (9:1), 20\% cholesterol, 5\% DSPE-PEG-biotin and $0.25 \%$ of fluorescent lipids. Our value of bending rigidity deduced from the critical angle $\theta_{c}$ is in the range of the expected value ${ }^{36}$.

The $t_{c}$ value defined by Eq. [7] depends on binder density, on their diffusion coefficient and also on the GUV radius. To compare $t_{c}$ values between different GUVs, we introduce a non-dimensional time $\tilde{t}=t$. $D / R_{v}^{2}$. Using for the diffusion coefficient of the binder $D=5 \mu \mathrm{m}^{2} \cdot \mathrm{sec}^{-1}$, measured in a similar case ${ }^{32}$, we found that $\widetilde{t_{c}}=0.4 \pm 0.1$ $(\mathrm{N}=6)$. By definition, $\widetilde{t_{c}}=\frac{\theta_{c}^{4}}{\varepsilon}=\frac{\gamma_{0}}{\Gamma_{0} \pi \kappa_{d}}$. As a consequence, knowing the value of the $\widetilde{t_{c}}$ and $\theta_{c}$ allows for the determination of $\varepsilon=0.09 \pm 0.03$. As $\varepsilon=2 U \Gamma_{0} / \gamma_{0}$, we can determine the density of binders $\Gamma_{0}$ on the glass surface. The values of $\gamma_{0}$ vary from one GUV to another and we are not able to accurately determine the membrane tension of each GUV in the present work. However, for GUVs formed in the same conditions and for similar lipid composition, typical values are in the range of $\gamma_{0}=10 \mu \mathrm{N} . \mathrm{m}^{-1}{ }^{37}$. Considering $U \approx 30 \mathrm{kT}$ for biotinstreptavidin link ${ }^{38}$, we can thus estimate the density of biotin binders $\Gamma_{0}=\frac{\varepsilon \gamma_{0}}{2 U}=4.10^{12} \mathrm{~m}^{-2}$. Considering the saturated concentration of biotin equal to $\Gamma_{\text {sat }} \approx 5.10^{15} \mathrm{~m}^{-2}{ }^{32}$, our surface treatment is thus in the dilute regime of binders $\Gamma_{0}=\Gamma_{\text {sat }} / 1000$.

The values of $\widetilde{t_{c}}$ highly depend on the binder density. For experiments performed in the opposite geometry (with streptavidin on the GUV) ${ }^{32}, \widetilde{t_{c}}$ was found to range from 0.3 to 5 when the adhesion conditions varied from $\Gamma_{\text {sat }}$ to $\Gamma_{\text {sat }} / 100$. Our value $\widetilde{t_{c}}=0.4 \pm 0.1$ is thus in the same range of values. 


\section{Water permeability of membrane and AQPO}

In order to determine the water permeability of membrane or that of AQPO, growth kinetics of the adhering patch has to be fitted with Eq. 13 to determine the exponential decay time $\tau$. According to Eq. 10, the decay time depends on the reduced volume of the shock $V r$, but also on the size of the GUV. To easily compare the characteristic decay from GUV to GUV, we plot the relative variation of adhering patch $\frac{A(t)-A_{f}}{A_{0}-A_{f}}$ as a function of the reduce time $\tilde{t}=t . D / R_{v}^{2}$ [14]. Typical decays are presented on Fig. 2C for membrane without AQPO (green), WtAQPO (red) and Tr-AQPO (blue) for a reduce volume $V_{r}=0.95$. The exponential decay time $\tilde{\tau}$ is equal to $10 \pm 0.5$ for membrane containing Wt-AQPO and is much faster than for the GUVs containing Tr-AQPO or without AQPO ( $\tilde{\tau}=36$ \pm 0.5 and $47 \pm 0.5$ respectively). We notice that the values of $\tilde{\tau}$ are always much larger than the values of $\widetilde{t_{c}}$ which was found to range from 0.3 to 0.5 , which was one of the assumption of our model. Indeed, the basic principle of our model relies on the fact that the characteristic time of adhesion due to binders is much faster than the characteristic time due to deflation. As a consequence, the GUV spreading during the osmotic shock is not limited by the diffusion of binder to the patch.

The membrane permeability deduced from the fit of the curves using Eq. 10 is presented on Fig. 2D for all batches of experiments. For membrane without AQPO, the permeability is found to be $P_{\text {memb }}=18 \pm 9 \mu \mathrm{m} / \mathrm{sec}$. The permeability of our specific membrane composition (EPC:EPA) (9:1) 80\% wt, 20\% cholesterol, 0.25\% Texas RedDHPE, and 5\% DSPE-PEG 2000 -Biotin lipids) has not been determined previously. However in case of membrane containing Egg PC only, the water permeability was found to be equal to $34 \mu \mathrm{m} \cdot \mathrm{sec}^{-1}{ }^{39}$. For DOPC membranes, this value ranges between 25 and $42 \mu \mathrm{m} \cdot \mathrm{sec}^{-1}$ and decreases to 5 to $7 \mu \mathrm{m} \cdot \mathrm{sec}^{-1}$ when cholesterol is added to the membrane ${ }^{21,40}$. Our value of $P_{\text {memb }}$ is thus compatible with the values previously published.

For membrane containing AQPO, the total membrane permeability (i.e. $P_{m e m b}+P_{A Q P O}$ ) is found to be equal to $66 \pm$ $15 \mu \mathrm{m} / \mathrm{sec}$ and $19 \pm 8 \mu \mathrm{m} / \mathrm{sec}$ for membrane containing Wt-AQPO and truncated AQPO respectively. Using the lipid membrane permeability value previously determined, the permeability due to AQPO is equal to $P_{W t-A Q P O}=48 \pm 15$ $\mu \mathrm{m} / \mathrm{sec}$ for full length AQPO and $P_{T r-A Q P O}=1 \pm 15 \mu \mathrm{m} / \mathrm{sec}$ for truncated AQPO. Since we measured the mean protein density in the GUVs for both protein types to be $1000 \pm 400 \mathrm{Wt}-\mathrm{AQPO} / \mu \mathrm{m}^{2}$ and $1300 \pm 350 \mathrm{Tr}-\mathrm{AQPO} / \mu \mathrm{m}^{2}$ respectively, one thus obtains the permeability of a single Wt-AQPO to be: $P_{\text {wt-AQPO }}=4.6 \pm 2.010^{-2} \mu^{3} \cdot \mathrm{s}^{-1}$ whereas for truncated AQPO, the permeability is found to be $P_{T r-A Q P O}=0.1 \pm 0.1 .10^{-2} \approx 0 \mu \mathrm{m}^{3} . \mathrm{s}^{-1}$. The water permeability of $A Q P$ is known to highly depend on its lipid environment. In a mixture of lipids containing POPC: POPG and cholesterol, the permeability of AQPO was found to vary from 3.5 in a mixture of PC:PG lipids (Phosphatidylcholine and Phosphatidylglycerol) to $0.5 \times 10^{-2} \mu \mathrm{m}^{3} \cdot \mathrm{s}^{-1}$ in SM:PG:Cholesterol (Sphigomyelin, Phosphatidylglycerol and Cholesterol) at $\mathrm{pH} 7.5^{11}$. It has also been found that lowering the $\mathrm{pH}$ from 8 to $\mathrm{pH} 6.5$ increases the efficiency of the AQPO by a factor 2 to $4{ }^{41,42}$. So our results obtained with a mixture of EPC, EPA and cholesterol at pH 6.5, are in perfect agreement with data previously published, which were performed on a population of liposomes. Molecular dynamics simulations have also been used to estimate the permeability of an AQPO monomer and it was found to be $0.28 \times 10^{-2} \mu \mathrm{m}^{3} . \mathrm{s}^{-1}$. As the AQPOs are composed of 4 monomers, this leads to a permeability of $1.1 \times 10^{-2} \mu^{3} . s^{-1}$ for a single AQPO ${ }^{41}$. As AQPO is known to be one of the less efficient water channels, the good agreement between our results and data previously published on water permeability of AQPO validate our experimental approach and technique.

Depending on its location, different functions have been reported for the AQPO. Wt-AQPOs that are located in the eyes lens cortex are known to be water-gated channels. With aging, the eye lens cells named fiber cells, go deeper 
inside the lens causing a proteolytic cleavage of the $\mathrm{N}$ - and C-terminal ends of the AQPO. The abundance of truncated AQPO in the lens increases with age ${ }^{41}$. The proteolytic cleavage of AQPO changes its function as it increases the propensity of AQPO to form end to end-junctions between two neighboring cells ${ }^{5}$. The capability of these truncated AQPOs to conduct water is still under debate. Two different studies where cRNA was injected in oocytes came to opposite conclusions on the capability of $\operatorname{Tr}$-AQPO to conduct water ${ }^{43}{ }^{44}$. The discrepancy probably arises from the difficulty to precisely determine the cell volume change in oocyte caused by an osmotic shock.

Using our method, we have been able to precisely determine the water permeability of Tr-AQPO inserted in GUV membranes. We unambiguously found that Tr-AQPOs produced by digestion of native AQPOs with chymotrypsin are not able to conduct water. Chymotrypsin digestion produce truncated AQPOs of $22 \mathrm{kDa}{ }^{7}$, which are known to form junctions. Note that in the present study, the AQPOs were incorporated in GUVs as single tetramers and since our study is performed on single vesicles, this assay cannot conclude about the capability of Tr-AQPO to form junctions.

Several methods have been developed to measure the water permeability of membrane proteins. But it is complicated either to know precisely the volume change due to non-spherical shape of liposomes or to determine the amount of protein expressed or incorporated in membranes. Here, we propose a new reliable way to measure the water permeability of single channels incorporated in membranes without any average on an ensemble of liposomes or any manipulation of vesicle. We have been able to accurately measure the water permeability of a low efficiency water channel. Our technique can therefore be used for water channels with an high water efficiency but probably at lower channel density in the membrane to ensure that the hypotheses of our model are still valid.

In the present work, we take advantage that hyper-osmotic shock causes an efflux of water throughout the AQPO water pore leading to the creation of an excess membrane area. The regulation of the cell volume and the cell membrane tension by the AQPs is important for many biological and mechanical processes. For instance, the AQPs are involved in cell migration. It has been shown that the AQP expression is increased at the front of migrating cell

${ }^{45}$, also in highly invasive cancer cells where AQPs are overexpressed ${ }^{46}$. Several models and hypotheses exist to understand the complex phenomena of cell migration. Among them, an "Osmotic Engine Model" has been proposed where osmotic shock and water permeation play a role in cell migration ${ }^{47}$. It might thus become important to have access to a robust in vitro assay such as our spreading assay to characterize more precisely the AQPs involved in these processes.

\section{Acknowledgements}

The authors would like to thanks Aurélie Bertin for the Cryo-EM image of the proteo-liposomes mixture. This work was supported by Institut Curie, Centre National de la Recherche Scientifique (CNRS), an "Interface physique biologie et chimie: Soutien à la prise de risque" CNRS grant, by the French National Research Agency through the "Investments for the Future" program (France-Biolmaging, ANR-10-INSB-04) and by the CelTisPhyBio Labex (N ANR-10-LBX-0038) part of the IDEX PSL (NANR-10-IDEX-0001-02 PSL). We also acknowledge the PICT-IBiSA (Institut Curie, Paris). A.B was supported by the "Ecole Doctorale Interdisciplinaire pour le vivant" (ED387). F.Q. was supported by the Fondation Pierre Gilles de Gennes (FPGG). P.B.'s group belongs to the CNRS consortium CellTiss. 


\section{References}

1. S. Balaz, Chemical Reviews, 2009, 109, 1793-1899.

2. A. S. Verkman, Annual Review of Medicine, 2012, 63, 303-316.

3. A. S. Verkman, Experimental Eye Research, 2003, 76, 137-143.

4. T. Gonen, Y. Cheng, P. Sliz, Y. Hiroaki, Y. Fujiyoshi, S. C. Harrison and T. Walz, Nature, 2005, 438, 633-638.

5. A. Engel, Y. Fujiyoshi, T. Gonen and T. Walz, Current Opinion in Structural Biology, 2008, 18, 229-235.

6. G. Chandy, G. A. Zampighi, M. Kreman and J. E. Hall, J. Membrane Biol., 1997, 159, 29-39.

T. Gonen, Y. Cheng, J. Kistler and T. Walz, Journal of molecular biology, 2004, 342, 1337-1345.

8. Huster, A. J. Jin, K. Arnold and K. Gawrisch, Biophysical journal, 1997, 73, 855-864.

9. J. Brahm, The Journal of General Physiology, 1982, 79, 791-819.

10. S. M. Mulders, G. M. Preston, P. M. T. Deen, W. B. Guggino, C. H. van OS and P. Agre, The Journal of Biological Chemistry, 1995, 270, 9010-9016.

11. J. Tong, J. T. Canty, M. M. Briggs and T. J. McIntosh, Experimental Eye Research, 2013, 113, 32-40.

12. M. Kumar, J. E. O. Habel, Y.-x. Shen, W. P. Meier and T. Walz, Journal of the American Chemical Society, 2012, 134, 18631-18637.

13. M. L. Zeidel, S. V. Ambudkar, B. L. Smith and P. Agre, Biochemistry, 1992, 31, 7436-7440.

14. J. C. Mathai, S. Tristram-Nagle, J. F. Nagle and M. L. Zeidel, The Journal of General Physiology, 2008, 131, 69-76.

15. J. Tong, Margaret M. Briggs and Thomas J. McIntosh, Biophysical journal, 2012, 103, 1899-1908.

16. M. Agemark, J. Kowal, W. Kukulski, K. Nordén, N. Gustavsson, U. Johanson, A. Engel and P. Kjellbom, Biochimica et Biophysica Acta (BBA) Biomembranes, 2012, 1818, 839-850.

17. K. Yakata, K. Tani and Y. Fujiyoshi, Journal of Structural Biology, 2011, 174, 315-320.

18. D. F. Savage and R. M. Stroud, Journal of molecular biology, 2007, 368, 607-617.

19. D. Kozono, X. Ding, I. Iwasaki, X. Meng, Y. Kamagata, P. Agre and Y. Kitagawa, Journal of Biological Chemistry, 2003, 278, $10649-10656$.

20. U. Seifert, K. Berndl and R. Lipowsky, Physical Review A, 1991, 44, 1182-1202.

21. W. Rawicz, B. A. Smith, T. J. McIntosh, S. A. Simon and E. Evans, Biophysical journal, 2008, 94, 4725-4736.

22. K. Olbrich, W. Rawicz, D. Needham and E. Evans, Biophysical journal, 2000, 79, 321-327.

23. R. Merkel, P. Nassoy, A. Leung, K. Ritchie and E. Evans, Nature, 1999, 397, 50-53.

24. F. Brochard-Wyart and P. G. de Gennes, Proceedings of the National Academy of Sciences, 2002, 99, 7854-7859.

25. P. G. de Gennes, P. H. Puech and F. Brochard-Wyart, Langmuir, 2003, 19, 7112-7119.

26. G. I. Bell, M. Dembo and P. Bongrand, Biophysical journal, 1984, 45, 1051-1064.

27. S. Aimon, J. Manzi, D. Schmidt, J. A. Poveda Larrosa, P. Bassereau and G. E. S. Toombes, PLoS ONE, 2011, 6, e25529.

28. L. Hasler, T. Walz, P. Tittmann, H. Gross, J. Kistler and A. Engel, Journal of molecular biology, 1998, 279, 855-864.

29. A. Berthaud, J. Manzi, J. Pérez and S. Mangenot, Journal of the American Chemical Society, 2012, 134, 10080-10088.

30. J. L. Rigaud, D. Levy, G. Mosser and O. Lambert, Eur Biophys J, 1998, 27, 305-319.

31. M. Garten, S. Aimon, P. Bassereau and G. E. S. Toombes, 2015, DOI: doi:10.3791/52281, e52281.

32. D. Cuvelier and P. Nassoy, Physical Review Letters, 2004, 93, 228101.

33. W. Helfrich, Zur Naturforschung, 1973, 28c, 693-703.

34. V. B. Shenoy and L. B. Freund, Proceedings of the National Academy of Sciences of the United States of America, 2005, 102, 3213-3218.

35. T. Bihr, S. Fenz, E. Sackmann, R. Merkel, U. Seifert, K. Sengupta and A.-S. Smith, Biophysical journal, 107, L33-L36.

36. W. Rawicz, K. C. Olbrich, T. McIntosh, D. Needham and E. Evans, Biophysical journal, 2000, 79, 328-339.

37. N. Borghi, O. Rossier and F. Brochard-Wyart, EPL (Europhysics Letters), 2003, 64, 837.

38. J. Wong, A. Chilkoti and V. T. Moy, Biomolecular Engineering, 1999, 16, 45-55.

39. A. Walter and J. Gutknecht, J. Membrane Biol., 1986, 90, 207-217.

40. A. Finkelstein and A. Cass, Nature, 1967, 216, 717-718.

41. M. $\varnothing$. Jensen, R. O. Dror, H. Xu, D. W. Borhani, I. T. Arkin, M. P. Eastwood and D. E. Shaw, Proceedings of the National Academy of Sciences, 2008, 105, 14430-14435.

42. K. L. Németh-Cahalan, K. Kalman and J. E. Hall, The Journal of General Physiology, 2004, 123, 573-580.

43. S. Sindhu Kumari and K. Varadaraj, Biochimica et Biophysica Acta (BBA) - General Subjects, 2014, 1840, 2862-2877.

44. L. E. Ball, M. Little, M. W. Nowak, D. L. Garland, R. K. Crouch and K. L. Schey, Investigative Ophthalmology \& Visual Science, 2003, 44, 4820-4828.

45. S. Saadoun, M. Papadopoulos, H. Watanabe, D. Yan, G. T. Manley and A. S. verkman, J. Cell. Sci, 2005, 118, 5961-5968.

46. A. S. Verkman, M. Hara-Chikuma and M. Papadopoulos, J Mol Med, 2008, 86, 523-529.

47. Kimberly M. Stroka, H. Jiang, S.-H. Chen, Z. Tong, D. Wirtz, Sean X. Sun and K. Konstantopoulos, Cell, 2014, 157, 611-623. 
A

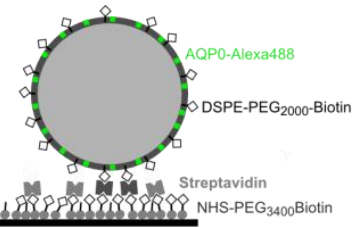

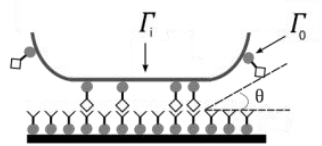

C
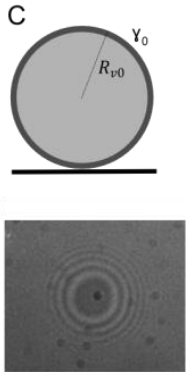

D
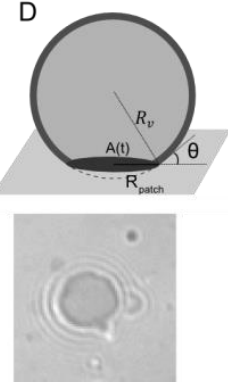

E

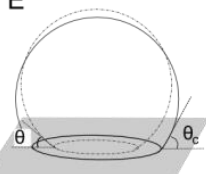

$\mathrm{F}$
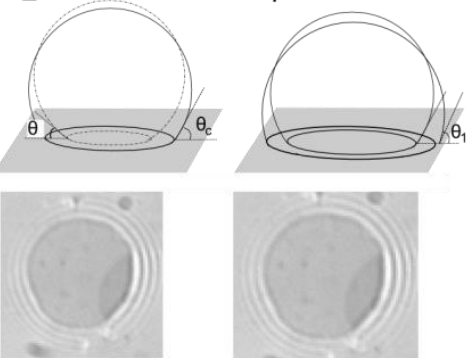

Figure 1: Principe of the RICM and osmotic shock experiments

(A) GUVs containing DSPE-PEG-biotin lipids, with or without AQPO sediment onto a functionalized surface coated with NHS-PEG-Biotin covered with streptavidin.

(B) When the vesicle adheres to the surface with a contact angle $\theta$, there is a diffusion of the binders from the vesicle to the patch. $\Gamma_{0}$ and $\Gamma_{\mathrm{i}}$ are respectively the binder surface osmotic pressure in the vesicle and in the patch.

Image C to F: Top is a schematic representation of the adhesion conditions. Bottom is the corresponding RICM image.

(C) GUVs are characterized by a membrane tension $\gamma_{0}$ and a radius $R_{v 0}$. GUVs approaching the surface are followed by RICM, even before the formation of the adhering patch

(D) GUVs adhering to the surface are characterized by the area of their adhesive patch $A(t)$, with a radius $R_{\text {patch }}(t)$. The apparent radius of the GUV is $R_{v o}$ and the contact angle between the GUV and the surface $\theta_{1}$.

(E) Before applying any osmotic shock, the GUVs spread on the surface until an equilibrium between the adhesion energy and the membrane tension energy is reached. The contact angle increases up to $\theta_{c}$. The spreading kinetics is followed by RICM (bottom).

An osmotic shock induces efflux of water out of the GUV, thus causing a decrease of surface tension. The excess free area is then transferred to the adhesion zone on the functionalized surface. The contact angle increases from $\theta_{\mathrm{c}}$ to $\theta_{1}$. 

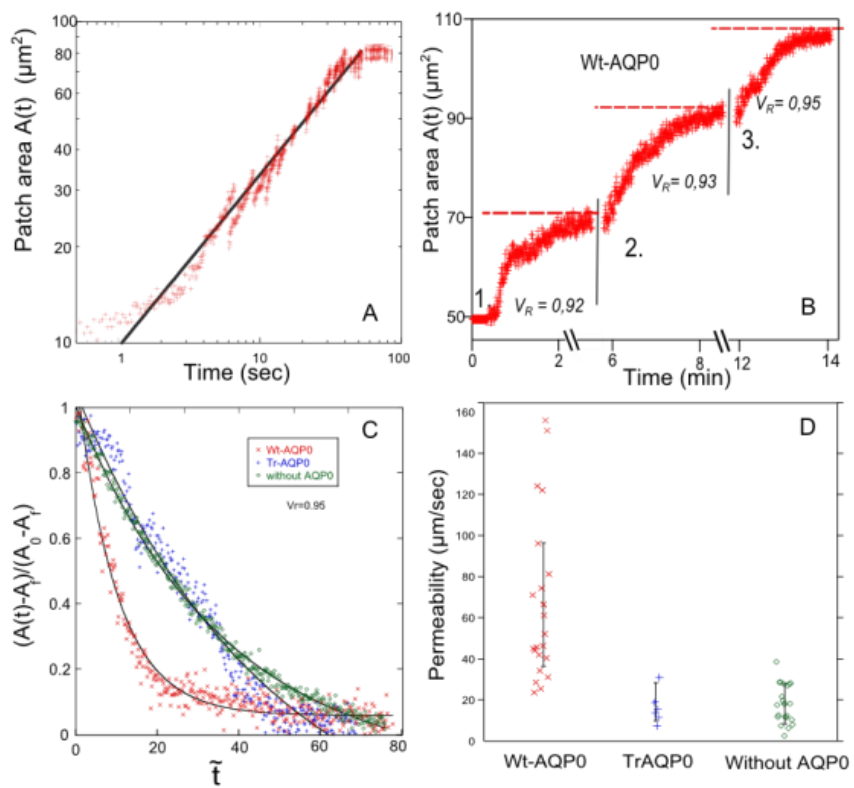

Figure 2: Kinetics of spreading during osmotic shocks.

(A) Variation of the adhesion patch area after that the GUV has contacted the substrate, in the absence of osmotic shock. The time dependence is fitted with a power Iaw $A=t^{t}$, with $n=0.53 \pm 0.02$ for $t<$ $50 \mathrm{sec}$ (straight black line on the graph). The existence of a plateau is the sign at ure of the absence of transit ory pores in the membrane.

(B) Variation of the patch area for a single GUV containing WtAQPO during successive osmotic shocks characterized by their reduced volume $V_{\text {,.. }}$

(c) The patch area $A(t)$ is normalized by the patch area before and after the application of the shock ( $A_{0}$ and $A_{f}$ respectively) $\frac{A(t)-A_{f}}{A_{0}-A_{f}}$ is then plotted as a function of the reduced time $\tilde{t}$ (defined by Eq. [14]) for GUVs without $A Q P 0(\triangle)$ or with Wt-AQP $0(x)$ or $\operatorname{Tr}-A Q P 0(+)$.

Curves are fitted with exponential decay (black continuous line). The exponential decay time $\tau=10 \pm 0.5$ for Wt-AQP0 $36 \pm 0.5$ for Tr-AQP0 and $47 \pm 0.5$ without $A Q P 0$.

Dat a presented on the graph are plotted for an osmotic shock $V_{i}=0.95$ for one GUV of each type.

Permeability of the membranes deduced from the kinetics of spreading for membranes with out $A Q P 0(\diamond)$ or with Wt-AQPO $(x)$ or Tr-AQPO $(t)$. 